\title{
Kazhdan-Lusztig polynomials of boolean elements
}

\author{
Pietro Mongelli ${ }^{1}$ | \\ ${ }^{1}$ Dipartimento di Matematica "G. Castelnuovo”, Università ”Sapienza” di Roma, P.le A. Moro 5, 00185 Roma, Italy
}

\begin{abstract}
We give closed combinatorial product formulas for Kazhdan-Lusztig poynomials and their parabolic analogue of type $q$ in the case of boolean elements, introduced in [M. Marietti, Boolean elements in Kazhdan-Lusztig theory, J. Algebra 295 (2006)], in Coxeter groups whose Coxeter graph is a tree. Such formulas involve Catalan numbers and use a combinatorial interpretation of the Coxeter graph of the group. In the case of classical Weyl groups, this combinatorial interpretation can be restated in terms of statistics of (signed) permutations. As an application of the formulas, we compute the intersection homology Poincaré polynomials of the Schubert varieties of boolean elements.

Résumé. Nous donnons des formules combinatories pour les polynômes de Kazhdan-Lusztig et leurs analogues parabolique de type $q$ pour les éléments booléens, introduite dans [M. Marietti, Boolean elements in Kazhdan-Lusztig theory, J. Algebra 295 (2006)], dans les groupes de Coxeter dont le graphe de Coxeter est un arbre. Ces formules utilisent les nombres de Catalan et une interprétation combinatoire des graphes du groupe de Coxeter. Dans le cas des groupes de Weyl classiques, cette interprétation combinatoire peut être reformulée en termes de statistiques de permutations avec signe. Avec ces formules, on peut calculer le polynôme de l'intersection homologie de Poincaré pour la variété de Schubert de booléen éléments.
\end{abstract}

Keywords: Coxeter groups, Kazhdan-Lusztig polynomials, Boolean elements, Poincaré polynomials

\section{Introduction}

In their fundamental paper Kazhdan and Lusztig (1979) defined, for every Coxeter group $W$, a family of polynomials, indexed by pairs of elements of $W$, which have become known as the Kazhdan-Lusztig polynomials of $W$ (see, e. g., (Humphreys, 1990, Chapter 7) or (Björner and Brenti, 2005, Chapter 5)). These polynomials play an important role in several areas of mathematics, including the algebraic geometry and topology of Schubert varieties and representation theory (see, e. g., (Björner and Brenti, 2005, Chapter 5), and the references cited there). In particular, their coefficients gives the dimensions of the intersection cohomology modules for Schubert varieties (see, e. g., Kazhdan and Lusztig (1980)).

In order to find a method for the computation of the dimensions of the intersection cohomology modules corresponding to Schubert varieties in $G / P$, where $P$ is a parabolic subgroup of the Kac-Moody group $G$,

\footnotetext{
${ }^{\dagger}$ This paper is part of the author's Ph.D. thesis written under the direction of Prof. F. Brenti at the Univ. "la Sapienza" of Rome, Italy.

1365-8050 @ 2013 Discrete Mathematics and Theoretical Computer Science (DMTCS), Nancy, France
} 
(Deodhar (1987)) introduced two parabolic analogues of these polynomials which correspond to the roots $x=q$ and $x=-1$ of the equation $x^{2}=q+(q-1) x$. These parabolic Kazhdan-Lusztig polynomials reduce to the ordinary ones for the trivial parabolic subgroup and are also related to them in other ways (see, e. g., Proposition 2.2 below). Besides these connections the parabolic polynomials also play a direct role in several areas including the theories of generalized Verma modules (Casian and Collingwood (1987)), tilting modules (Soergel (1997a), Soergel (1997b)) and Macdonald polynomials(Haglund et al. (2005a), Haglund et al. (2005b)).

The purpose of this work is to give explicit combinatorial product formulas for all (parabolic and ordinary) Kazhdan-Lusztig polynomials indexed by pairs of boolean elements (see Section 2 for the definition) in all Coxeter groups whose Coxeter graph is a tree. Our results show that all such polynomials have nonnegative coefficients, conjectured by Kazhdan and Lusztig (1979), and give a combinatorial interpretation of them in terms of Catalan numbers and the Coxeter graph of the group. In the case of classical Weyl groups, this combinatorial interpretation can be restated in terms of excedances and other statistics of (signed) permutations. Our results also confirm a conjecure of Brenti on the parabolic Kazhdan-Lusztig polynomials of type $q$ (see Corollary 3.3 below).

\section{Definitions, notation and preliminaries}

We let $\mathbb{P}:=\{1,2,3, \ldots\}, \mathbb{N}:=\mathbb{P} \cup\{0\}, \mathbb{Z}:=\mathbb{N} \cup\{-1,-2, \ldots\}$. For all $m, n \in \mathbb{Z}, m \leq n$ we set $[m, n]:=\{m, m+1, \ldots, n\}$ and $[n]:=[1, n]$. Given a set $A$ we denote by $\# A$ its cardinality.

We follow (Stanley, 1997, Chapter 3) for poset notation and terminology. In particular, given a poset $(P, \leq)$ and $u, v \in P$ we let $[u, v]:=\{w \in P \mid u \leq w \leq v\}$ and call this an interval of $P$. We say that $v$ covers $u$, denoted $u \triangleleft v$ (or, equivalently, that $u$ is covered by $v$ ) if \# $[u, v]=2$.

We follow Humphreys (1990) for general Coxeter groups notation and terminology. Given a Coxeter system $(W, S)$ and $u \in W$ we denote by $l(u)$ the length of $u$ in $W$, with respect to $S$, i. e. the minimal length of words $s_{i_{1}} \cdots s_{i_{k}}=u$ whose alphabet is $S$ (such minimal words are called reduced). Given $u, v \in W$ we denote by $l(u, v)=l(v)-l(u)$. We let $D_{R}(u):=\{s \in S \mid l(u s)<l(u)\}$ the set of the right descents of $u, D_{L}(u):=\{s \in S \mid l(s u)<l(u)\}$ the set of the left descents of $u$ and we denote by $\epsilon$ the identity of $W$. Given $J \subseteq S$ we let $W_{J}$ the parabolic subgroup generated by $J$ and

$$
W^{J}:=\{u \in W \mid l(s u)>l(u) \text { for all } s \in J\}
$$

Note that $W^{\emptyset}=W$ (the above definition is a little bit different from the classical one given in (Björner and Brenti 2005, Definition 2.4.2)). If $W_{J}$ is finite, then we denote by $w_{0}(J)$ its longest element. We will always assume that $W^{J}$ is partially ordered by Bruhat order. Recall (see e.g. (Humphreys, 1990, Chapter 5.9 and 5.10)) that this means that $x \leq y$ if and only if for one reduced word of $y$ (equivalently for all) there exists a subword that is a reduced word of $x$. Given $u, v \in W^{J}, u \leq v$ we let

$$
[u, v]^{J}:=\left\{w \in W^{J} \mid u \leq w \leq v\right\}
$$

and $[u, v]:=[u, v]^{\emptyset}$.

For $J \subseteq S, x \in\{-1, q\}$ and $u, v \in W^{J}$ we denote by $P_{u, v}^{J, x}(q)$ the parabolic Kazhdan-Lusztig polynomials in $W^{J}$ of type $x$ (we refer the reader to Deodhar (1987) for the definitions of these polynomials, see also Proposition 2.2 below). We denote by $P_{u, v}(q)$ the ordinary Kazhdan-Lusztig polynomials. 
For $u, v \in W^{J}$ let $\mu_{J, q}(u, v)$ be the coefficient of $q^{\frac{1}{2}(l(u, v)-1)}$ in $P_{u, v}^{J, q}(q)$ (so $\mu_{J, q}(u, v)=0$ when $l(v)-l(u)$ is even). It is well known that if $u, v \in W^{J}$ then $\mu_{J, q}(u, v)=\mu(u, v)$, the coefficient of $q^{\frac{1}{2}(l(u, v)-1)}$ in $P_{u, v}(q)$ (see Corollary 2.1 below). The following result is due to Deodhar, and we refer the reader to Deodhar (1987) for its proof.

Proposition 2.1 Let $(W, S)$ be a Coxeter system, $J \subseteq S$, and $u, v \in W^{J}, u \leq v$. Then for each $s \in D_{R}(v)$ we have that

$$
P_{u, v}^{J, q}(q)=\widetilde{P}_{u, v}-\widetilde{M}_{u, v}
$$

where

$$
\widetilde{P}_{u, v}= \begin{cases}P_{u s, v s}^{J, q}+q P_{u, v s}^{J, q} & \text { if } u s<u \\ q P_{u s, v s}^{J, q}+P_{u, v s}^{J, q} & \text { if } u<u s \in W^{J} \\ 0 & \text { if } u<u s \notin W^{J}\end{cases}
$$

and

$$
\widetilde{M}_{u, v}=\sum_{u \leq w<v s \mid w s<w} \mu(w, v s) q^{\frac{l(w, v)}{2}} P_{u, w}^{J, q}(q) .
$$

The parabolic Kazhdan-Lusztig polynomials are related to their ordinary counterparts in several ways, including the following one, which may be taken as their definition in most cases.

Proposition 2.2 Let $(W, S)$ be a Coxeter system, $J \subseteq S$ and $u, v \in W^{J}$. Then we have that

$$
P_{u, v}^{J, q}(q)=\sum_{w \in W_{J}}(-1)^{l(w)} P_{w u, v}(q) .
$$

Moreover, if $W_{J}$ is finite, then

$$
P_{u, v}^{J,-1}(q)=P_{w_{0}(J) u, w_{0}(J) v}(q) .
$$

A proof of this result can be found in Deodhar (1987) (see Proposition 3.4, and Remark 3.8). Since for all $w \in W_{J}$ and $u \in W^{J}$ we have $l(w u)=l(w)+l(u)$ by (Björner and Brenti 2005. Proposition 2.4.4), then the degree of $P_{w u, v}(q)$ in Proposition 2.2 is less than $\frac{1}{2}(l(u, v)-1)$ except when $w=\epsilon$. Therefore we have

Corollary 2.1 For any $J \subseteq S$ and $u, v \in W^{J}$ we have

$$
\mu_{J, q}(u, v)=\mu(u, v) .
$$

Proposition 2.3 Let $(W, S)$ a Coxeter system and $J \subseteq S$. Let $u, v \in W^{J}$ and $s \in D_{R}(v)$.

a) If us $\notin W^{J}$ then $P_{u, v}^{J, q}(q)=0$;

b) if $u s \in W^{J}$ then $P_{u s, v}^{J, q}(q)=P_{u, v}^{J, q}(q)$;

c) if $\mu(u, v) \neq 0$ then $D_{R}(v) \subseteq D_{R}(u)$ and $D_{L}(v) \subseteq D_{L}(u)$.

In the rest of the paper we will consider parabolic Kazhdan-Lusztig polynomials of type $q$. Therefore we will write $P_{u, v}^{J}$ instead of $P_{u, v}^{J, q}$.

Let $(W, S)$ be any Coxeter system and $t$ be a reflection in $W$. Following Marietti (Marietti (2002), Marietti (2006) and Marietti (2010)), we say that $t$ is a boolean reflection if it admits a boolean expression, 
which is, by definition, a reduced expression of the form $s_{1} \cdots s_{n-1} s_{n} s_{n-1} \cdots s_{1}$ with $s_{k} \in S$, for all $k \in\{1, \ldots n\}$ and $s_{i} \neq s_{j}$ if $i \neq j$. We say that $u \in W$ is a boolean element if $u$ is smaller than a boolean reflection in the Bruhat order. Let $\bar{v}$ be a reduced word of a boolean element and $s \in S$, we denote by $\bar{v}(s)$ the number of occurrences of $s$ in $\bar{v}$.

Given a Coxeter system $(W, S)$, the Coxeter graph of $W$ is a graph whose vertex set is $S$ and two vertices $s, s^{\prime}$ are joined by an edge if $s s^{\prime} \neq s^{\prime} s$. We label this edge with $m\left(s, s^{\prime}\right)$, the smallest positive integer such that $\left(s s^{\prime}\right)^{m\left(s, s^{\prime}\right)}=\epsilon\left(m\left(s, s^{\prime}\right)=\infty\right.$ if there is no such integer). We say that $W$ is a treeCoxeter group if its Coxeter graph is a tree.

fai o nuova sezione o breve intro For any generator $s_{i} \in S$ we denote by $S^{i}=S \backslash\left\{s_{i}\right\}$ and by $\operatorname{com}\left(s_{i}\right)$ the subset of $S$ which contains all elements commuting with $s_{i}$ different from $s_{i}$.

Lemma 2.1 Let $u, v \in W^{J}$ such that $s_{i} u, s_{i} v \in W_{S^{i}}^{J}$ (i. e. there exist reduced words for $u, v$ starting with $s_{i}$ and with no other occurrences of $\left.s_{i}\right)$. Then

$$
P_{u, v}^{J}=P_{s_{i} u, s_{i} v}^{J \cap \operatorname{com}_{v}\left(s_{i}\right)} .
$$

Lemma 2.2 Let $u, v \in W^{J}$ be such that $u, s_{i} v \in W_{S^{i}}$ (i. e. there are no occurrences of $s_{i}$ in any reduced expression of $u$ and $s_{i} v$ ). Then

$$
P_{u, v}^{J}= \begin{cases}P_{u, s_{i} v}^{J} & \text { if } s_{i} v \in W^{J} \\ 0 & \text { otherwise }\end{cases}
$$

We now introduce a family of numbers which are used in the next section. The Catalan triangle is a triangle of numbers formed in the same manner as Pascal's triangle, except that no number may appear on the left of the first element (see (OEI, sequence A008313)).

$\begin{array}{ccccccccccc}1 & & & & & & & & \\ & 1 & & & & & & & & \\ 1 & & 1 & & & & & & & \\ & 2 & & 1 & & & & & & \\ 2 & & 3 & & 1 & & & & & \\ & 5 & & 4 & & 1 & & & & \\ 5 & & 9 & & 5 & & 1 & & & \\ & 14 & & 14 & & 6 & & 1 & & \\ 14 & & 28 & & 20 & & 7 & & 1 & \\ & 42 & & 48 & & 27 & & 8 & & 1\end{array}$

Let $h \geq 1$. We set

$$
f_{h}(q)=\sum_{i=0}^{\left[\frac{h}{2}\right]} C(h, i) q^{\left[\frac{h}{2}\right]-i}
$$

where $[h]$ denotes the integer part of $h$ and $C(h, i)$ is the $i$-th number in the $h$-th row (here we start the enumeration from 0 ). For example $f_{4}(q)=2 q^{2}+3 q+1 ; f_{7}(q)=14 q^{3}+14 q^{2}+6 q+1$. Note that in the first column we find the classical Catalan numbers (see (OEI, sequence A008313) for details). 


\section{Parabolic Kazhdan-Lusztig polynomials}

Let $(W, S)$ be a tree-Coxeter group. Let $t=s_{i_{1}} \cdots s_{i_{n-1}} s_{i_{n}} s_{i_{n-1}} \cdots s_{i_{1}}$ be a boolean reflection. Consider the Coxeter graph $G$ and represent it as a rooted tree with root the vertex corresponding to the generator $s_{i_{n}}$. In this paper all the roots will be depicted on the right of their graphs. In Figure 1 we give the Coxeter graph of the affine Weyl group $\widetilde{D}_{11}$.

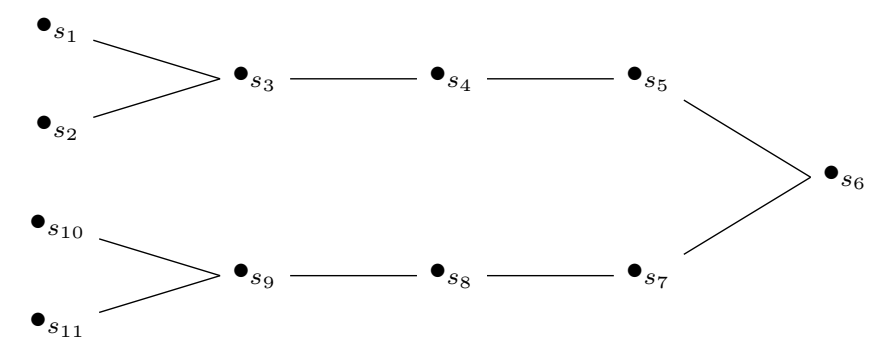

Fig. 1: The Coxeter graph of $\widetilde{D}_{11}$ with root $s_{6}$, corresponding to the reflection $t=$ $s_{1} s_{2} \cdots s_{5} s_{10} s_{11} s_{9} s_{8} s_{7} s_{6} s_{7} s_{8} s_{9} s_{11} s_{10} s_{5} \ldots s_{2} s_{1}$.

According to such rooted graph we say that $s_{j}$ is on the right (respectively on the left) of $s_{i}$ if and only if there exists an edge joining them and the only path from $s_{i}$ to $s_{n}$ contains $s_{j}$.

Let $w$ be a word in the alphabet $S$ and $s \in S$. We denote by $w(s)$ the number of occurrences of $s$ in $w$. Let $u, v \in W$ be such that $u, v \leq t$. Let $\bar{u}, \bar{v}$ be the unique reduced expressions of $u, v$ satisfying the following properties

- $\bar{v}$ is a subword of $s_{1} \cdots, s_{n-1} s_{n} s_{n-1} \cdots s_{1}$ and if $i$ is such that $\bar{v}\left(s_{i}\right)=1$ and $\bar{v}\left(s_{j}\right)=0$, where $s_{j}$ is the only element on the right of $s_{i}$, then we choose the subword with $s_{i}$ in the leftmost admissible position;

- $\bar{u}$ is a subword of $\bar{v}$ and if $i$ is such that $\bar{u}\left(s_{i}\right)=1$ and $\bar{u}\left(s_{j}\right)=0$, we apply the same above rule.

Here we give an example. Let $t=s_{1} s_{2} \ldots s_{5} s_{10} s_{11} s_{9} s_{8} s_{7} s_{6} s_{7} s_{8} s_{9} s_{11} s_{10} s_{5} \ldots s_{2} s_{1}$ in $\widetilde{D}_{11}$, see Figure 1 Let $v=s_{4} s_{5} s_{10} s_{11} s_{6} s_{7} s_{8} s_{9} s_{5} s_{4} s_{2} s_{1}$ and $u=s_{8} s_{6} s_{1}$ then $\bar{v}=s_{1} s_{2} s_{4} s_{5} s_{10} s_{11} s_{6} s_{7} s_{8} s_{9} s_{5} s_{4}$ and $\bar{u}=s_{1} s_{6} s_{8}$.

Now we give a graphical representation of the pair $(\bar{v}, \bar{u})$. We start from the rooted tree of the Coxeter graph and we substitute for each vertex a table with one column and two rows. In the first row we write $\bar{v}\left(s_{j}\right)\left(s_{j}\right.$ is the element associated to the vertex); in the second row we write $\bar{u}\left(s_{j}\right)$. In the case $\bar{v}\left(s_{j}\right)=1$, it is possible that $s_{j}$ is on the left or on the right of $s_{n}$ (the root) as subword of $t$. We distinguish the two cases by writing $1_{l}$ if $s_{j}$ is on the left of $s_{n}$, and $1_{r}$ otherwise. By convention we write $1_{l}$ in the root $s_{n}$ if $\bar{v}\left(s_{n}\right) \neq 0$. We apply the same rule to the second row. Moreover, in the first row, we use capital letter $R$ instead of $r$ if the second row of the column to the right does not contain 0 .

We mark the column corresponding to $s_{j}$ with $\circ$ if $j \in J$ and with $\times$ if $j \notin J$. Finally, if a vertex $s_{j}$ has only one vertex on the left then we write the two corresponding columns in same table. In Figure 2 we give the graphical representation of the pair $(\bar{v}, \bar{u})$ in $\widetilde{D}_{11}$, with $J=\left\{s_{5}, s_{7}\right\}$. 


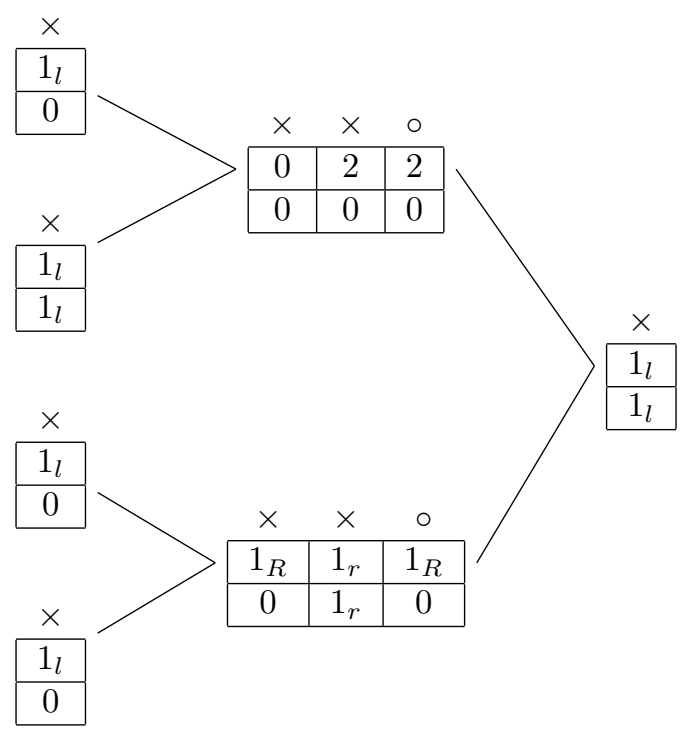

Fig. 2: Diagram of $\left(\bar{v}=s_{1} s_{2} s_{4} s_{5} s_{11} s_{10} s_{6} s_{7} s_{8} s_{9} s_{5} s_{4}, \bar{u} s_{1} s_{6} s_{8}\right)$ in $\widetilde{D}_{11}$.

In the sequel a symbol $*$ denotes the possibility to have arbitrary entries in the cell. A symbol such as $A_{l}$, $\varnothing$, etc. means that the value in the cell is not $1_{l}, 0$, etc. Moreover we will be interested in subdiagrams of such representations, i. e. diagrams obtained by deleting one or more columns. Since the order of the tables from top to bottom is not important (while the order from left to right is fundamental), we use the following notation

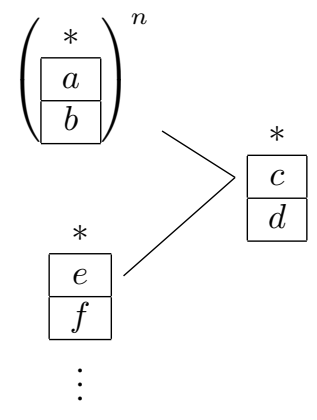

to mean

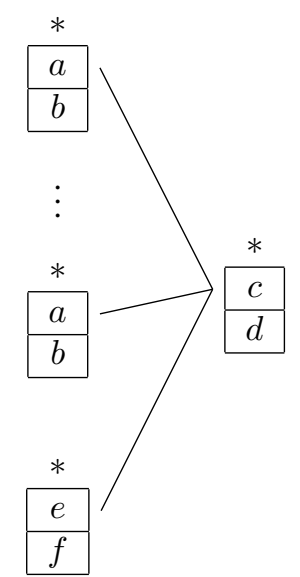


where the column with entries $a, b$ is repeated $n$ times. Now we give all the definitions necessary to Theorem 3.1

Given a pair $(\bar{v}, \bar{u})$ in $W$, we let $a_{h}(\bar{u}, \bar{v})$ be the number of subdiagrams in the diagram of $(\bar{u}, \bar{v})$ of one of the following type:

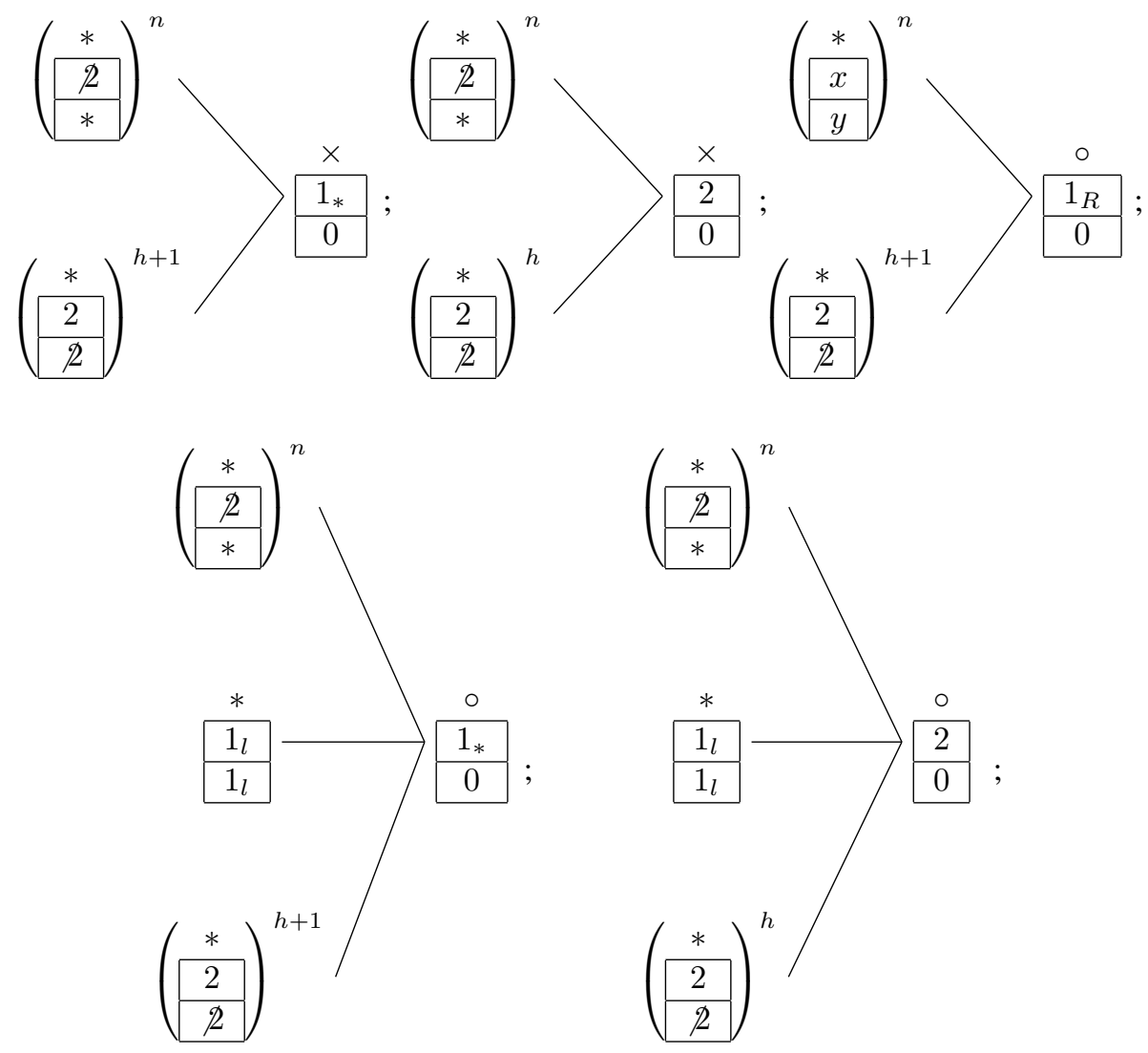

We define $b_{h}(\bar{u}, \bar{v})$ be the number of subdiagrams in the diagram of $(\bar{u}, \bar{v})$ of one of the following type:
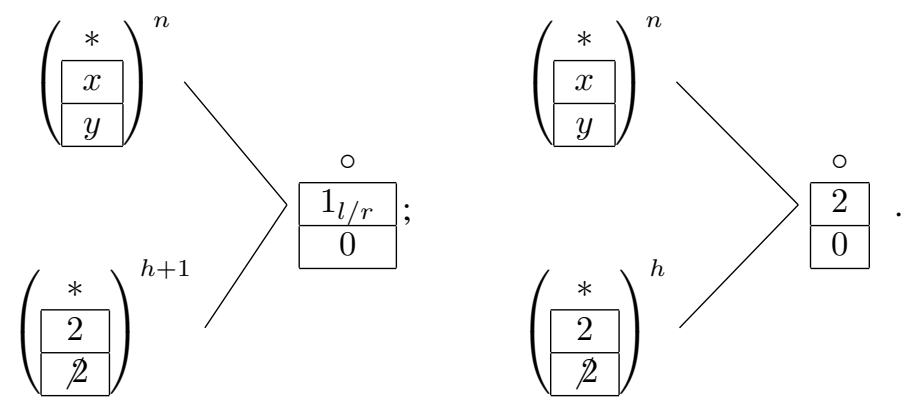
We set $c(\bar{u}, \bar{v})$ be the number of subdiagrams in the diagram of $(\bar{u}, \bar{v})$ of one of the following type:

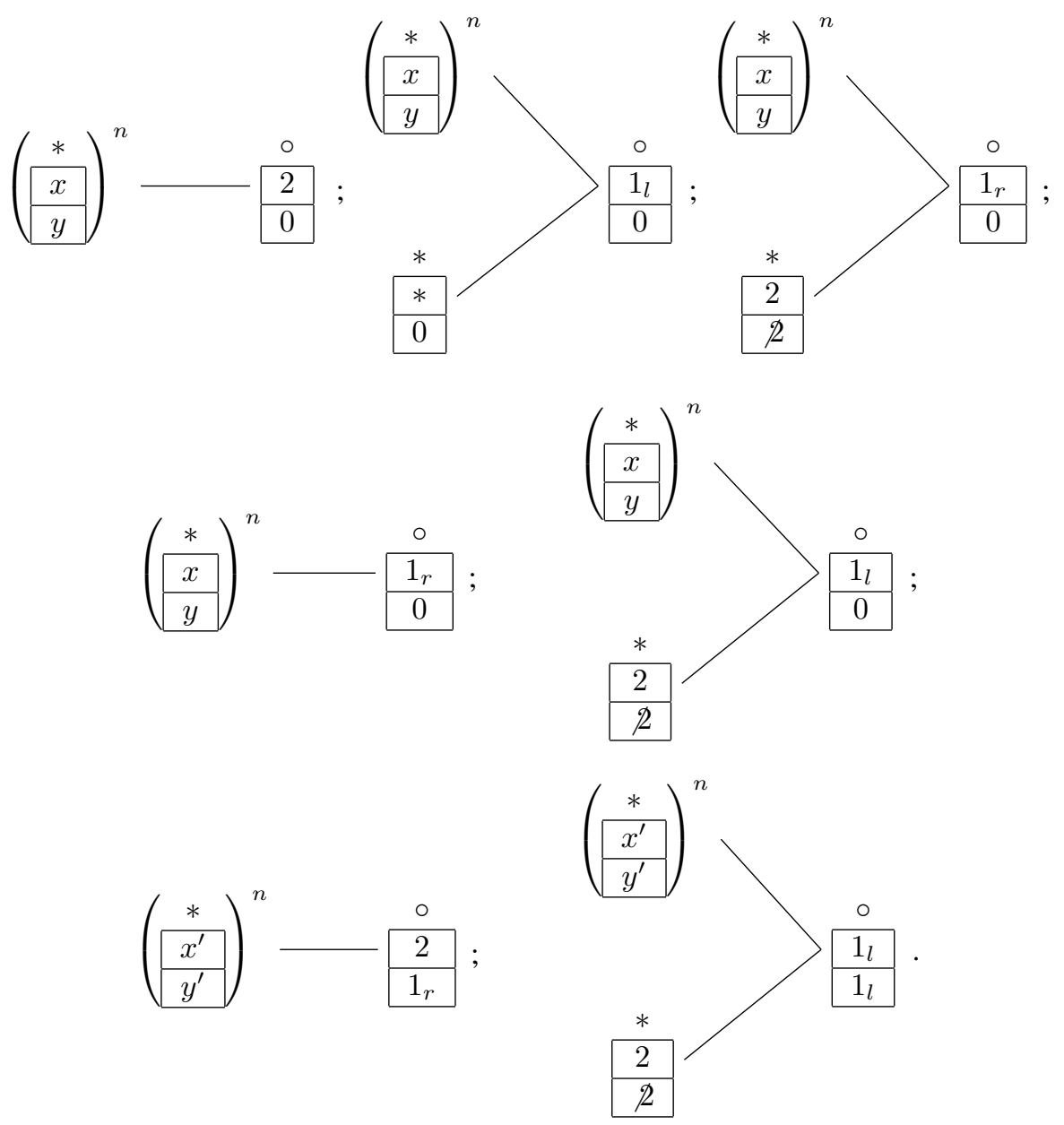

Finally, we set $c^{\prime}(\bar{u}, \bar{v})$ be the number of subdiagrams of the diagram of $(\bar{u}, \bar{v})$ of the following type:

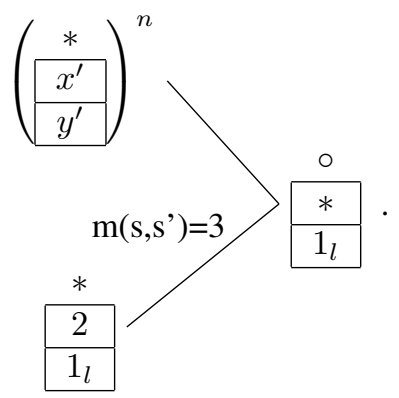


In all previous diagrams $n$ is an arbitrary non-negative integer and $(x, y) \in P_{1},\left(x^{\prime}, y^{\prime}\right) \in P_{1} \cup P_{2}$ with $P_{1}=\left\{\left(1_{l}, 0\right),\left(1_{r}, 0\right),\left(1_{r}, 1_{r}\right),\left(2,1_{r}\right)\right\}, P_{2}=\left\{\left(1_{R}, 0\right),\left(1_{R}, 1_{r}\right),(2,0)\right\}$. In each diagram $(x, y),\left(x^{\prime}, y^{\prime}\right)$, $(\not 2, *)$ or $(2, \not 2)$ are not necessarily the same pair for all $n \geq 0$ (or $h \geq 0$ ) columns. We can now state the main result of this work.

Theorem 3.1 Let $J \subseteq S, u, v \in W^{J}$ and set $\bar{c}(\bar{u}, \bar{v})=c(\bar{u}, \bar{v})+c^{\prime}(\bar{u}, \bar{v})$. We have

$$
P_{u, v}^{J}(q)= \begin{cases}\prod_{h \geq 1} f_{h+1}^{a_{h}}\left(f_{h+1}-1\right)^{b_{h}} & \text { if } \bar{c}(\bar{u}, \bar{v})=0 \\ 0 & \text { otherwise }\end{cases}
$$

Corollary 3.1 Let $J \subseteq S, u, v \in W^{J}$ with $l(v)-l(u) \geq 3$ odd. Then $\mu(u, v) \neq 0$ if and only if the entries in each column of the diagram of $(\bar{u}, \bar{v})$ are equal except for exactly one subdiagram which is

$$
\left(\begin{array}{c}
* \\
\hline 2 \\
\hline 1_{l}
\end{array}\right)^{h+1}-\begin{aligned}
& * \\
& \varnothing \\
& \hline 0
\end{aligned} \text { or }\left(\begin{array}{c}
* \\
\hline 2 \\
\hline 1_{l}
\end{array}\right)^{h}-\begin{array}{|l|l|l|}
* & \multicolumn{2}{|c|}{*} \\
\hline 2 & \ldots & 2 \\
\hline 0 & \ldots & 0 \\
\hline
\end{array}
$$

In this case $\mu(u, v)=C\left(\left[\frac{h+1}{2}\right]\right)$, the $\left[\frac{h+1}{2}\right]$-th Catalan number.

In the case of the classical Kazhdan-Lusztig polynomials, Theorem 3.1 becomes much simpler.

Corollary 3.2 Let $W$ be a tree-Coxeter group and $u, v \in W$ be boolean elements. Then $P_{u, v}(q)=$ $\prod_{h \geq 1} f_{h+1}^{a_{h}}$, where $a_{h}$ is defined before Theorem 3.1 .

For example, the Kazhdan-Lusztig polynomial of the pair $(u, v)$ depicted in Figure 2 is $P_{u, v}^{J}=f_{2}(q)-$ $1=q$, since $a_{h}=0$ for all $h \geq 0, b_{1}=1$ and $b_{h}=0$ for all $h \neq 1$.

Remark 3.1 Theorem 3.1 implies result in (Marietti. 2010 Theorem 5.2).

We give the following easy consequence of Theorem 3.1 which proves, in the case of boolean elements, a conjecture of Brenti (private communication).

Corollary 3.3 Let $I \subseteq J$ and $u, v \in W^{J}$. Then

$$
P_{u, v}^{J}(q) \leq P_{u, v}^{I}(q)
$$

in the coefficientwise comparison.

\section{cita solo atilde}

\section{Poincaré polynomials}

Given $v \in W$, let $F_{v}(q)=\sum_{u \leq v} q^{l(v)} P_{u, v}$. It is well known that, if $W$ is any finite Coxeter or affine Weyl group, $F_{v}(q)$ is the intersection homology Poincaré polynomial of the Schubert variety indexed by $v$ (see Kazhdan and Lusztig (1980)). In this section we compute the Poincaré polynomial for any boolean element in a Coxeter group whose Coxeter graph is a tree with at most one vertex having more than two adjacent vertices (such groups include all classical finite Coxeter and affine Weyl groups except $\widetilde{A}_{n}$ and $\left.\widetilde{D}_{n}\right)$. 
Let $v \in W$ be a boolean element and consider the diagram of $\left(\epsilon_{W}, \bar{v}\right)$. For convenience we will not depict the second row of each column which is always 0 and we omit all symbols $\times$. We will call it the diagram of $v$.

Let $v$ be a boolean element and let $s$ be the element of $S$ associated to one of the leftmost vertices in the diagram of $v$. We set $F_{v, s}=\sum q^{l(v)} P_{u, v}$ where the sum runs over all elements $u \leq v$ such that $\bar{u}(s) \neq 0$ and $F_{v, s}^{0}=\sum q^{l(v)} P_{u, v}$ where the sum runs over all elements $u \leq v$ such that $\bar{u}(s)=0$.

Now consider a diagram $d$. Delete all entries equal to 0 and delete all edges whose left vertex is not a cell containing 2 . Let $d_{1}, \ldots, d_{k}$ be the remaining connected components. We refer to them as the essential components of $d$.

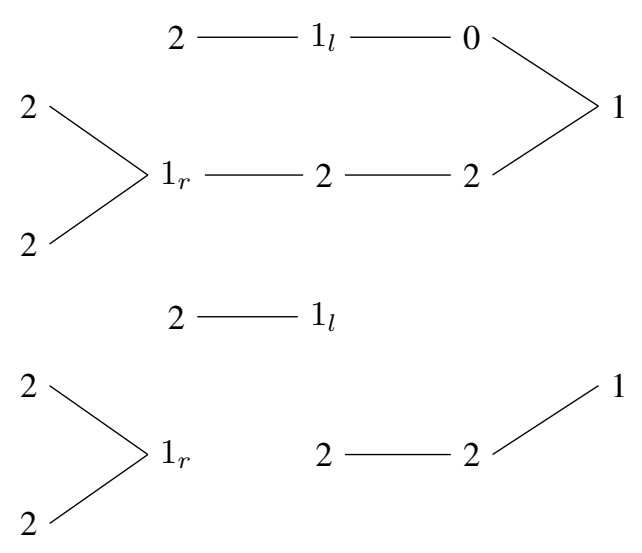

Fig. 3: A diagram and its essential components.

Lemma 4.1 Let $v \in W$ be a boolean element and let $d$ be the diagram of $\bar{v}$. Let $d_{1}, \ldots, d_{k}$ be the essential components of the diagram $d$ and $v_{1}, \ldots, v_{k}$ be the boolean reflections corresponding to $d_{1}, \ldots, d_{k}$. Then

$$
F_{v}(q)=\prod_{i=1}^{k} F_{v_{i}}(q) .
$$

Proposition 4.1 Let $W$ be a Coxeter group such that its Coxeter graph is a tree and all vertices except at most one have degree less than 3 . Denote with $w$ such exceptional vertex. Let $v \in W$ be a boolean element. Then

$$
F_{v}(q)=\left(1+q+q^{2}\right)^{k-1}\left(q(1+q)^{h+1}+f_{h}(q)\right)(1+q)^{l(v)-2 k-h-2},
$$

where $k$ is the number of essential components of the diagram $d$ of $v$ with at least two vertices and $h$ is the number of entries equal to 2 in the adjacent cells of $w$ (also consider the cell on the right).

The formula is also true when there is no vertex of degree greater than 2: in this case let $w$ be any vertex of degree 2. 


\section{Acknowledgements}

At the end of the manuscript, right before the bibliography you might want to place an acknowledgement. This can be easily done by using the command \acknowledgements as you can see here.

\section{References}

The On-Line Encyclopedia of Integer Sequences. published electronically at http://oeis.org.

A. Björner and F. Brenti. Combinatorics of Coxeter Groups. Number 231 in Graduates Texts in Mathematics. Springer-Verlag, New York, 2005.

L. Casian and D. Collingwood. The Kazhdan-Lusztig conjecture for generalized Verma modules. Math. Zeit., 195:581-600, 1987.

V. Deodhar. On some geometric aspects of Bruhat orderings. II. The parabolic analogue of KazhdanLusztig polynomials. J. Algebra, 111:483-506, 1987.

J. Haglund, M. Haiman, and N. Loehr. A combinatorial formula for Macdonald polynomials. J. Amer. Math. Soc., 18:735-761, 2005a.

J. Haglund, M. Haiman, N. Loehr, J. Remmel, and A. Ulyanov. A combinatorial formula for the character of the diagonal coinvariants. Duke Math. J., 126:195-232, 2005b.

J. Humphreys. Reflection groups and Coxeter Groups. Cambridge Studies in Advanced Mathematics, no. 29, Cambridge Univ. Press, Cambridge, 1990.

D. Kazhdan and G. Lusztig. Representations of Coxeter Groups and Hecke Algebras. Invent. Math., 53: 165-184, 1979.

D. Kazhdan and G. Lusztig. Schubert varieties and Poincaré duality. Proc. Sympos. Pure Math., 36: 185-203, 1980.

M. Marietti. Closed product formulas for certain R-polynomials. Europ. J. of Combin., 23:57-62, 2002.

M. Marietti. Boolean elements in Kazhdan-Lusztig theory. J. Algebra, 295:1-26, 2006.

M. Marietti. Parabolic Kazhdan-Lusztig and $R$-polynomials for Boolean elements in the symmetric group. Europ. J. of Combin., 31:908-924, 2010.

W. Soergel. Kazhdan-Lusztig polynomials and a combinatoric for tilting modules. Represent. Theory, 1: 83-114, 1997a.

W. Soergel. Character formulas for tilting modules over Kac-Moody algebras. Represent. Theory, 1: 115-132, 1997b.

R. P. Stanley. Enumerative combinatorics, volume 1. Cambridge University Press, Cambridge, 1997. 
